\title{
Investigation of numbers and motility of spermatozoa in reproductively active and socially suppressed males of two eusocial African mole-rats, the naked mole-rat (Heterocephalus glaber) and the Damaraland mole-rat (Cryptomys damarensis)
}

\author{
C. G. Faulkes ${ }^{1}$, S. N. Trowell ${ }^{2}$, J. U. M. Jarvis and \\ N. C. Bennett ${ }^{3}$ \\ ${ }^{1}$ Reproductive Biology Group, Institute of Zoology, Zoological Society of London, Regent's Park, \\ London NW1 4RY, UK; ${ }^{2}$ King's College London, London W8 7AH, UK; and ${ }^{3}$ Department of \\ Zoology, University of Cape Town, Rondebosch 7700, Cape Town, South Africa
}

\begin{abstract}
Reproductive tracts and spermatozoa from reproductively active and reproductively suppressed non-breeding males from two species of eusocial African mole-rats Cryptomys damarensis and Heterocephalus glaber were examined. In two captive colonies of Heterocephalus glaber, reproductive tracts from seven non-breeding males removed from their colonies, and housed singly for 5-6 weeks to cause reproductive activation, were compared with reproductive tracts from seven non-breeding males. The body weight of the separated, reproductively active males increased significantly $(P<0.01)$, and the mean testis weights relative to body weight of the reproductively active males were significantly larger $(P<0.05)$ than those of non-breeding males. The number of spermatozoa, in one half of the reproductive tract, was higher in active males than in non-breeding males (mean \pm SEM: $8.59 \times 10^{6} \pm 2.69 \times 10^{6}$ versus $1.78 \times 10^{6} \pm 1.43 \times 10^{6}$, respectively; $P<0.05$ ). In addition, six of the seven reproductively active males, but only two of seven non-breeding males, had motile spermatozoa. A total of 28 wild Cryptomys damarensis from two colonies were examined in the field. The testis weights relative to body weight of breeding males $(n=7)$ were higher than those of non-breeding males $(n=19 ; P<0.01)$, but the number of spermatozoa did not differ significantly between the two groups $\left(0.13 \times 10^{6} \pm 0.06 \times 10^{6}, n=7\right.$ versus $0.29 \times 10^{6} \pm 0.14 \times 10^{6}, n=21$, respectively). Breeding and non-breeding males produced similar numbers of motile spermatozoa. These results suggest that socially induced reproductive suppression of males differs in these two species of African mole-rats.
\end{abstract}

\section{Introduction}

The African mole-rats, family Bathyergidae, are a unique taxon of subterranean rodents in that they exhibit the widest range of social systems of any mammal. These range from solitary, for example the Cape mole-rat (Georychus capensis), to the colonial common mole-rat (Cryptomys hottentotus), and the eusocial Damaraland mole-rat (Cryptomys damarensis) and naked molerat (Heterocephalus glaber). The two last mentioned species are highly social and characterized by overlapping generations, and clear reproductive and behavioural divisions of labour within their colonies (Jarvis, 1981; Jarvis and Bennett, 1991).

The naked mole-rat inhabits arid regions of Kenya, Ethiopia and Somalia, and lives in extensive underground burrow systems that commonly contain $40-90$, but sometimes up to 300 , individuals. In these colonies one female, the queen, is

Received 4 May 1993 reproductively active, and she mates with $1-3$ specific individuals, known as 'breeding males' (Jarvis, 1981; Brett, 1991; Lacey and Sherman, 1991). Non-breeding females are reproductively quiescent and do not ovulate (Faulkes et al., 1990a, b). A suppression of reproductive hormones is also apparent in non-breeding males. Concentrations of urinary testosterone and plasma $\mathrm{LH}$ are higher in breeding males than in nonbreeding males, although spermatogenesis occurs in the latter, and spermatozoa are present in the vas deferens (Faulkes and Abbott, 1991; Faulkes et al, 1991). As in females, non-breeding male naked mole-rats rapidly become reproductively active on removal from social cues which cause suppression. If separated from their colonies, and either housed singly, or paired with a female, concentrations of plasma $\mathrm{LH}$ and urinary testosterone in non-breeding males increase to concentrations comparable to those of breeding males (Faulkes and Abbott, 1991). Naked mole-rats in the wild and in captivity breed continuously throughout the year, with oestrus in the queen occurring 8-10 
days post partum. During oestrus, which may last from 2 to $24 \mathrm{~h}$, the queen appears to initiate courtship and solicit mating with one or both breeding males (Jarvis, 1991).

The Damaraland mole-rat is found in semi-arid regions of southwestern and central Africa (Smithers, 1983). As in the naked mole-rat, breeding is restricted to one female, and she copulates with one or two specific 'breeding males'. Colonies generally contain 12-25 animals (Bennett and Jarvis, 1988; Jarvis and Bennett, 1991). Breeding animals are the largest and most dominant individuals within the colony (Jacobs et al., 1991), and reproduction occurs continuously throughout the year (Jarvis and Bennett, 1991). As with the naked mole-rat, ovulation of non-breeding females in a Damaraland mole-rat colony is inhibited (Bennett et al., in press). In contrast to the naked mole-rat, in non-breeding males plasma $\mathrm{LH}$ and urinary testosterone concentrations do not differ from those of breeding males (Bennett et al,, in press). Thus, while a socially induced suppression of reproductive physiology is apparent in females, in the male Damaraland mole-rat, suppression may be due entirely to an inhibition of reproductive behaviour.

The possible effects of reproductive suppression on male fertility were measured by determining the numbers of spermatozoa and sperm motility of reproductively active and socially suppressed individuals of both species.

\section{Methods}

\section{Animals}

A total of 14 males was studied in two captive colonies of naked mole-rats. One group of seven non-breeders ranging in body weight from 23.2 to $31.0 \mathrm{~g}$ (mean; $26.2 \pm 1.0 \mathrm{~g}$ ) were killed and their reproductive tracts were removed as described below. A second group of seven non-breeders was removed from their colonies, housed singly for 5-6 weeks to allow them to become reproductively active 'breeding males' (Faulkes and Abbott, 1991); they were then killed and their reproductive tracts were removed, as described below. Although they had not copulated with a queen in a colony situation, the hormonal profiles of these 'reproductively active' males resemble those of breeding males before mating (Faulkes and Abbott, 1991). Body weights of these males at the time of removal from their colony were similar to those of the non-breeder group in this study, ranging from 21.7 to $30.8 \mathrm{~g}$ (mean \pm SEM: $26.9 \pm 1.2 \mathrm{~g}$ ). The mean body weight of naked mole-rats in our captive colonies was about $30.0 \mathrm{~g}$.

A total of 28 male Damaraland mole-rats (seven breeders and 21 non-breeders) from two complete wild colonies was used in this study. In one colony containing a total of 41 animals, six breeding males were identified. The second colony of 11 individuals contained one breeding male. Animals were caught in the vicinity of Oddballs Camp in the Okavango Delta, Botswana $\left(19^{\circ} 30^{\prime} \mathrm{S} 23^{\circ} 6^{\prime} \mathrm{E}\right)$ using Hickman livetraps (Hickman, 1979). Breeding males were distinguished from non-breeders by the presence of 'bulging testes' situated abdominally in inguinal pockets. Previous studies have correlated this distinguishing anatomical feature with sexual activity, mating behaviour and thus breeding status (Bennett and Jarvis, 1988).

\section{Removal of reproductive tract tissue}

Animals were killed by i.p. injection of $0.4 \mathrm{ml}$ of Euthatal (Rhône Merieux Ltd, Harlow) (naked mole-rats) or inhalation of halothane (Damaraland mole-rats); reproductive tracts were removed with the aid of a dissecting microscope, and placed into BWW medium (Biggers et al, 1971) containing $4 \mathrm{mg} \mathrm{BSA} \mathrm{ml}{ }^{-1}$ (naked mole-rats), or RPMI 1640 medium (Gibco BRL, Uxbridge) with glutamine and $20 \mathrm{mmol}$ Hepes $1^{-1}$ (Damaraland mole-rats). Tracts from each animal were cut just below the junction of the vasa deferentia to produce left and right halves consisting of testis, epididymis, vas deferens, seminal vesicle and sperm storage sac. One half of the reproductive tract (chosen at random) was fixed in $0.05 \mathrm{~mol}$ phosphate-buffered glutaraldehyde $\mathrm{I}^{-1}(2 \%)$ at $4^{\circ} \mathrm{C}$ before it was weighed. The remaining half of the tract was used for determination of the number of spermatozoa and assessment of their motility as described below.

\section{Histology}

After fixation in glutaraldehyde, tissue samples were postfixed in $1 \%$ osmium tetroxide in $0.05 \mathrm{~mol}$ phosphate buffer $1^{-1}$ for $5 \mathrm{~h}$ at $4^{\circ} \mathrm{C}$, then dehydrated through $90 \%$ and $100 \%$ ethanol, propylene oxide, and embedded in epoxy resin (Epon-Araldite: Fisons, Loughborough). Sections of $1 \mu \mathrm{m}$ were cut using a Reichert-Jung Ultracut E ultramicrotome, and stained with a solution of $1 \%$ toluidine blue and $1 \%$ borax. Photomicrographs were taken on Ilford Pan-F film using an Olympus $\mathrm{BH} 2$ photomicroscope.

\section{Sperm motility}

Spermatozoa from naked mole-rats were squeezed from the distal portion (including the vas deferens) of the remaining side of each reproductive tract, into $100 \mu \mathrm{l}$ of BWW media. A drop of the sperm suspension was then placed in a Helber counting chamber $(20 \mu \mathrm{l}$ depth), and examined on a preheated microscope stage at $32^{\circ} \mathrm{C}$ with negative high phase contrast optics. Sperm motion analysis was performed using a computerized image analysis system (Hobson Sperm Tracker; Sense and Vision, Electronic Systems Ltd, Sheffield). The average speed of spermatozoa $\left(\mu \mathrm{m} \mathrm{s}^{-1}\right)$ was calculated as the mean of the total track distance travelled by a spermatozoon divided by the total track time. An estimate was made of sperm motility. Approximately 100 spermatozoa were counted and motility scored using the following scale: $0-25 \%$ motile, $1 ; 26-50 \%$ motile, 2 ; $51-75 \%$ motile, $3 ; 76-100 \%$ motile, 4 .

For Damaraland mole-rats caught in the field, sperm motility was assessed at the site of capture by placing a drop of medium containing spermatozoa, squeezed from the distal vas deferens, onto a glass microscope slide prewarmed to approximately $30^{\circ} \mathrm{C}$. Scores of sperm motility were made as described for the naked mole-rats.

\section{Number of spermatozoa}

The remaining spermatozoa contained within the tract from each animal were squeezed out into fixative, using fine forceps 

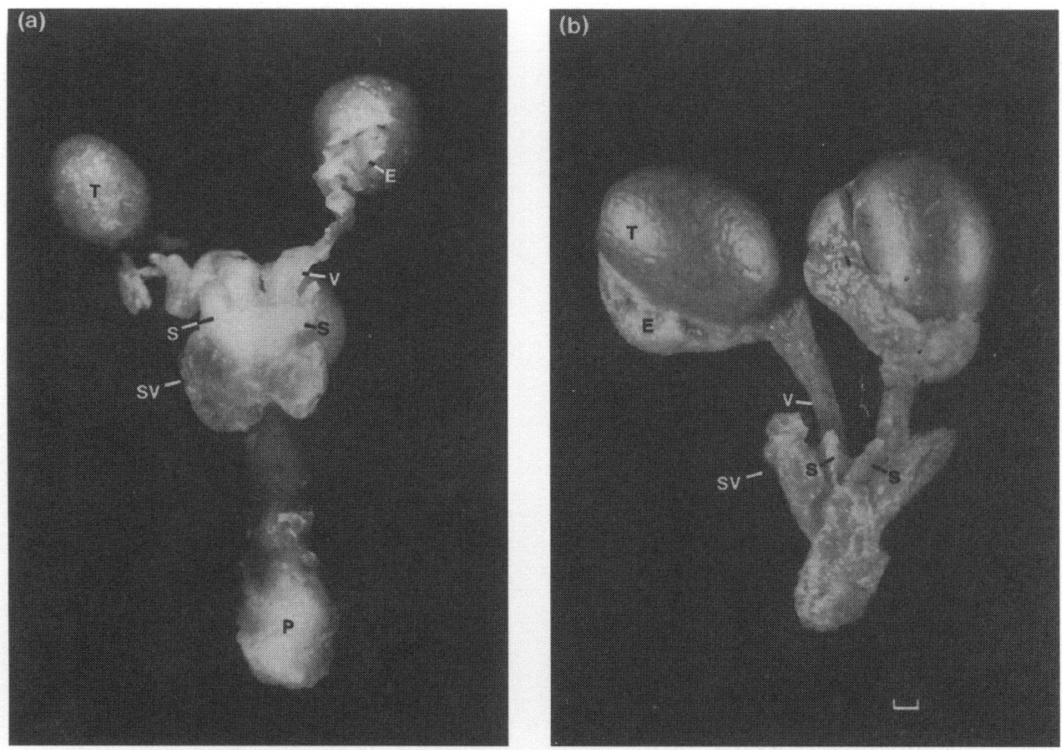

Fig. 1. Reproductive tract and testes of breeding males in (a) the naked mole-rat, and (b) the Damaraland mole-rat. $T$ : testis; E: epididymis; V: vas deferens; $S$ : sperm storage sac; SV: seminal vesicle; $P$ : penis.

and with the aid of a dissecting microscope, and combined with the respective sperm samples used for measurements of motility. To ensure removal of all the spermatozoa, the tracts were cut into smaller sections, and squeezing continued until no more spermatozoa were expelled. Volumes for each sample were adjusted to give a total of $250 \mu \mathrm{l}$ fixative. The number of spermatozoa in each sample was determined using a haemocytometer.

\section{Statistical analysis}

All comparisons of parameters measured in breeding and non-breeding males were performed using Student's $t$ test, except estimates of percentage of motile spermatozoa, which were analysed using Mann-Whitney's $U$ test.

\section{Results}

\section{Body weights}

The body weights of non-breeding male naked mole-rats separated from their parent colonies and housed singly for 5-6 weeks increased significantly to $32.8 \pm 2.0 \mathrm{~g}$ (mean percentage increase: $22.4 \pm 5.3 ; n=7 ; \quad P<0.01$ ). Thus reproductively active males had a larger body weight than did non-breeding males at the time their reproductive tracts were examined (32.8 $\pm 2.0 \mathrm{~g}$ versus $26.2 \pm 1.0 \mathrm{~g} ; P<0.05$ ).

The body weights of Damaraland breeding males $(n=7)$ caught in the field were significantly greater than those of non-breeding males $(n=21)$ : $194.5 \pm 11.3 \mathrm{~g}$ versus $116.0 \pm 7.7 \mathrm{~g}$, respectively $(P<0.01)$.

\section{Anatomy of reproductive tract}

In both species of mole-rat, the reproductive tract and testes lie within the abdominal cavity. The epididymis is relatively small and convoluted at the proximal end. The duct becomes less convoluted distally, where it emerges into the vas deferens (Fig. 1a, b). The tracts of reproductively active naked mole-rats possessed a prominent pair of sperm storage sacs situated at the junction of the vasa deferentia, containing large numbers of spermatozoa (Fig. 2a). These organs were also present in non-breeding males, but were small and underdeveloped in most individuals (Fig. 2b). In the Damaraland mole-rat, similar structures were present in both breeding and non-breeding males, but at the time of dissection, none of the animals had a sac that was swollen with spermatozoa, and in that respect they resembled the non-breeding naked mole-rats (Fig. 2c, d).

\section{Testis and seminal vesicle weights}

Mean testis weights of reproductively active male naked mole-rats were significantly greater relative to body weight than those of non-breeding males: $1.06 \pm 0.33 \mathrm{mg} \mathrm{g}^{-1}(n=7)$ versus $0.59 \pm 0.05 \mathrm{mg} \mathrm{g}^{-1}(n=7)$, respectively $(P<0.05)$. There was no significant difference in mean seminal vesicle weights, relative to body weight, between non-breeding and reproductively active males: $0.25 \pm 0.06 \mathrm{mg} \mathrm{g}^{-1}$ versus $0.33 \pm 0.08 \mathrm{mg} \mathrm{g}^{-1}$, respectively.

In Damaraland mole-rats, testis weights of breeding males $(n=7)$ were greater relative to body weight than those of non-breeding males $(n=19): 0.91 \pm 0.07 \mathrm{mg} \mathrm{g}^{-1}$ versus $0.64 \pm 0.03 \mathrm{mg} \mathrm{g}^{-1}$, respectively $(P<0.01)$. Seminal vesicle weights, relative to body weight, were not significantly different between the two groups (breeding males: $0.30 \pm 0.04 \mathrm{mg}$ $\mathrm{g}^{-1}, n=7$; non-breeding males: $0.32 \pm 0.04 \mathrm{mg} \mathrm{g}^{-1}, n=10$ ).

\section{Sperm motility}

Six of seven separated male naked mole-rats became reproductively active and had motile spermatozoa, compared with 

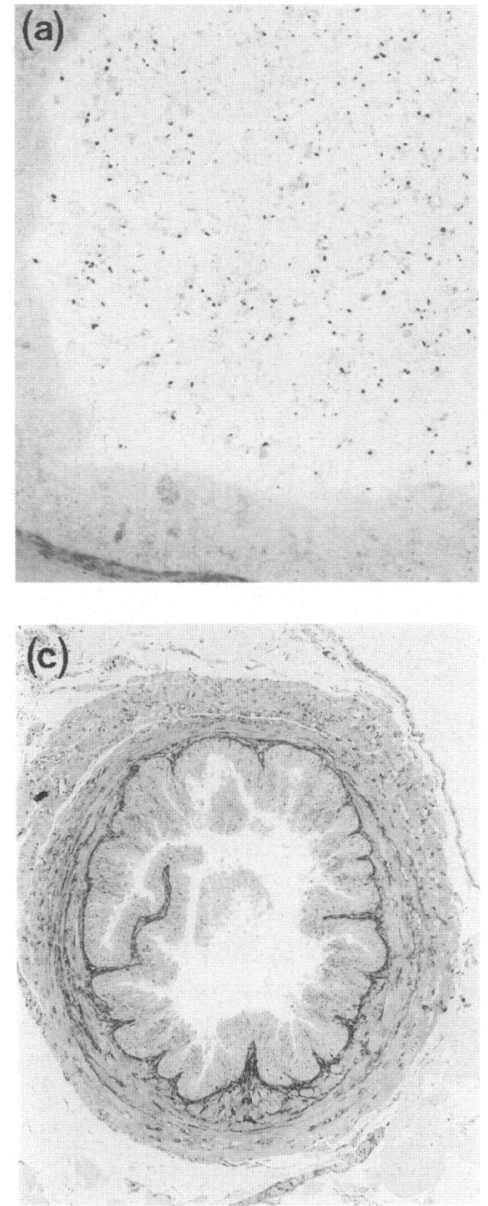

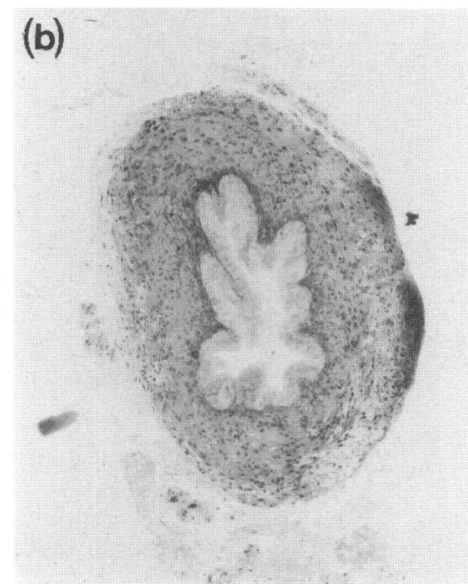

(d)

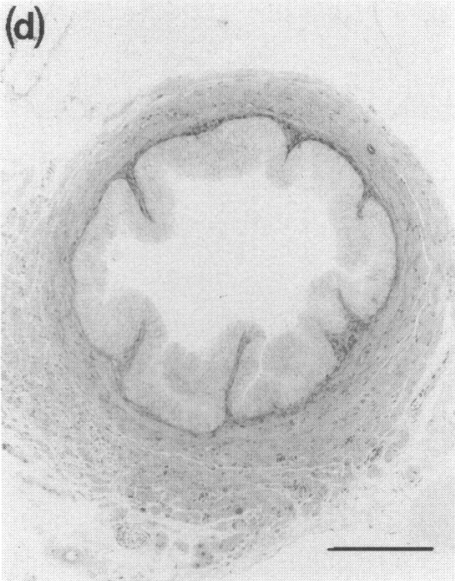

Fig. 2. Transverse sections through the sperm storage sac of (a) a breeding, and (b) a non-breeding male naked mole-rat, and similar structures in (c) a breeding, and (d) a non-breeding male Damaraland mole-rat. The enlarged size of this structure and presence of many spermatozoa are clearly seen in the breeding male naked mole-rat (a). Scale bar represents $50 \mu \mathrm{m}$.

only two out of seven non-breeding males. Both the number and speed $\left(\mu \mathrm{m} \mathrm{s}^{-1}\right)$ of motile spermatozoa tracked from these two non-breeding males were comparable to those of active males (Fig. 3).

Combining data from non-breeding and reproductively active males, there was no significant correlation between numbers of spermatozoa and body weight $(r=0.532, P>0.05)$ or sperm speed and body weight $(r=0.289, P>0.05)$. Estimates of the percentage of motile spermatozoa in non-breeding and reproductively active males were significantly different $(P<0.05$; Fig. 4a).

In contrast to naked mole-rats, in Damaraland mole-rats captured in the field, both breeding and non-breeding males had motile spermatozoa. Estimates of the percentage of motile spermatozoa were not significantly different between the two reproductive groups (Fig. $4 \mathrm{~b}$ ).

\section{Numbers of spermatozoa}

In naked mole-rats, total numbers of spermatozoa present in one side of the reproductive tract of reproductively active males $(n=7)$ were significantly higher than those of nonbreeding males $(n=7): 8.59 \times 10^{6} \pm 2.69 \times 10^{6}$ versus $1.78 \times 10^{6} \pm 1.43 \times 10^{6}$, respectively $(P<0.05)$.

In Damaraland mole-rats, numbers of spermatozoa did not differ significantly between breeding $(n=7)$ and nonbreeding $(n=21)$ males: $0.13 \times 10^{6} \pm 0.06 \times 10^{6}$ versus $0.29 \times 10^{6} \pm 0.14 \times 10^{6}$, respectively.

\section{Discussion}

This study shows clear species differences in the mechanisms of reproductive suppression in non-breeding male mole-rats. In Damaraland mole-rats, there was no apparent suppression of sperm production or motility in the reproductive tract of non-breeding males, supporting previous endocrine studies that found no differences in urinary testosterone (Bennett $e t$ al., in press), plasma $\mathrm{LH}$, or $\mathrm{LH}$ responses to exogenous $\mathrm{GnRH}$ (Bennett et al., 1993) between breeding and non-breeding males. The larger relative testes masses seen in breeding males compared with non-breeding males was consistent with the 


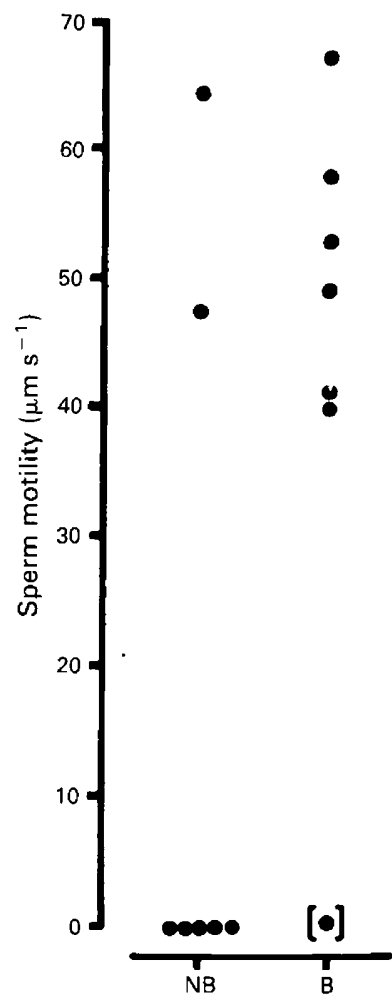

Fig. 3. Measurements of sperm motility in seven breeding (B) and seven non-breeding $(\mathrm{NB})$ naked mole-rats. One of the separated males [-] failed to become reproductively active for unknown reasons.

observation that these individuals have 'bulging testes' situated in inguinal pockets, enabling them to be differentiated morphologically from non-breeders. However, these larger testes did not appear to produce more spermatozoa and it is possible that the extra mass is interstitial tissue, although this was not determined. Differences between the amount of interstitial tissue in breeding and non-breeding males have been noted in naked mole-rats (Faulkes, 1990), which are unusual in having large numbers of interstitial cells (Fawcett et al., 1973). While one of the colonies caught in the field contained a single breeding male, in the second colony of 41 individuals, six males were characterized as breeders from the presence of abdominally bulging testes. This was the largest colony of Damaraland mole-rats so far captured, and may explain the presence of more breeding males. Previously, reproduction has been shown to be restricted to one or two males in colonies containing up to 25 animals (Bennett and Jarvis, 1988; Jarvis and Bennett, 1991).

In naked mole-rats, clear hormonal differences between breeding and non-breeding male naked mole-rats have been shown (Faulkes and Abbott, 1991; Faulkes et al., 1991). The results reported here support the endocrine data and suggest that in non-breeding males, suppression of reproductive hormones may correlate with reduced fertility, because most produce significantly fewer, non-motile spermatozoa, than do reproductively active males. In some non-breeding males, urinary testosterone and plasma LH concentrations are relatively high (Faulkes et al., 1991), and this finding is consistent with the observation that two non-breeding males examined in (a)

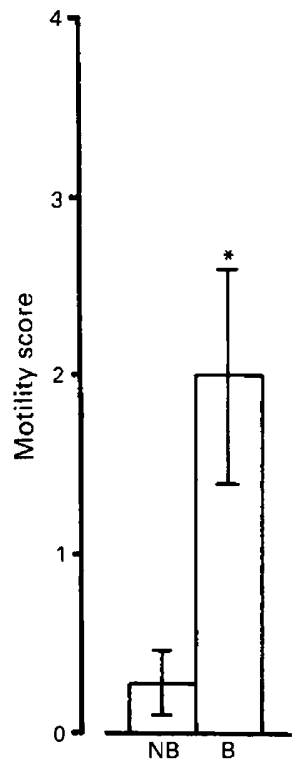

(b)

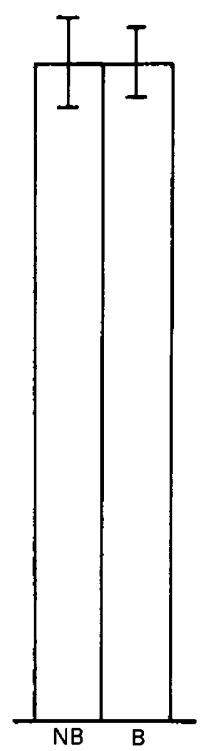

Fig. 4. Mean ( \pm SEM) percentage motility scores in breeding $(B)$ and non-breeding (NB) males in (a) naked mole-rats (NB: $n=7$; $\mathrm{B}: n=7$; ${ }^{*} P<0.05$ versus NB), and (b) in Damaraland mole-rats (NB: $n=18 ; B$ : $n=6$ ). Motility score - 1: $0-25 \%$ motile; 2: $26-50 \%$ motile; 3 : 51-75\% motile; $4: 76-100 \%$ motile.

this study had motile spermatozoa. Among non-breeders in a colony, there may be a number of individuals that are reproductively active and potentially fertile, should the breeding queen solicit them for mating. Lack of correlation between body weight and either numbers of spermatozoa or sperm motility suggests that attainment of a certain body size is not a prerequisite for reproductive activation.

Removal of non-breeding males from suppression by separation from their colonies led to increases in body weight, relative testis weight, and production of greater numbers of motile spermatozoa. These changes occurred within 5-6 weeks after removal from the social cues within their colonies that induce suppression. Plasma LH and urinary testosterone increase significantly after separation of males from the colony (Faulkes and Abbott, 1991). The enlargement of sperm storage sacs after separation and reproductive activation in males suggests that the development of these structures may be androgen dependent. It would be interesting to investigate whether sperm storage in breeding males in colonies varies over the ovarian cycle of the queen, in synchrony with fluctuating testosterone concentrations in the male, which peak before oestrus (Faulkes and Abbott, 1991). Histologically, the sperm storage sacs resembled the vas deferens, and therefore anatomically the former may be derived from the latter. The functional significance of these storage sacs in breeding male naked mole-rats is unclear, but presumably they are necessary adaptations, because the relatively small epididymis and vas deferens in this species may provide limited sperm storage capacity. Although similar structures were apparent in both breeding and non-breeding Damaraland mole-rats, in no cases were the sacs found to be engorged with spermatozoa, as in singly-housed, reproductively active naked mole-rats. It is 
possible that, before mating with an oestrous female, increased storage of spermatozoa could occur in breeding male Damaraland mole-rats, but at the time the animals were captured and examined none was in this condition.

Behavioural cues from the breeding queen naked mole-rat have been implicated in suppression of reproduction in nonbreeding males and females (Faulkes and Abbott, 1993), and in controlling reproduction in breeding males (Faulkes and Abbott, 1991). These studies show that in this species, social cues, predominantly in the form of behavioural interactions, can be physiologically translated into reduced and impaired sperm production. Such a block to reproduction is rare among male mammals that are socially suppressed. In male dwarf mongoose, for example, there are no differences in testosterone between breeding and non-breeding males, and reproductive suppression apparently results from an exclusion from mating of these males (Creel et al., 1992). Lack of a distinct physiological suppression of reproduction in male Damaraland mole-rats, like non-breeding mongooses, is more typical of a socially suppressed male mammal, and may reflect differences in mating strategies and dispersal patterns compared with those of naked mole-rats.

Recent molecular phylogenetic investigations of mitochondrial DNA sequence variation have suggested that naked mole-rats separated from a common ancestor early in the evolutionary history of the family Bathyergidae, and that Damaraland mole-rats are divergent and have a relatively independent evolutionary origin (Allard and Honeycutt, 1992). Such studies suggest that highly social behaviour and reproductive suppression has evolved independently, but in parallel, giving rise to different physiological mechanisms of suppression in the two species.

The authors thank M. J. Llovet and the laboratory animal staff at the Institute of Zoology for care and maintenance of the naked mole-rats, A. S. I. Loudon, B. R. Brinklow and W. V. Holt for criticism of the manuscript, T. Dennett and M. J. Walton for preparation of the figures and $\mathrm{H}$. $\mathrm{O}^{\prime}$ Brien for technical help with histology and photomicrography. They also thank K. Schrumpf, P. Bell, N. Snow, G. Aguilar and C. Faulkes for assistance in the field work. This work was supported by grants from the University of Cape Town, and the Foundation for Research and Development (JUMJ and NCB). The field work was supported by a grant from the National Geographic Society.

\section{References}

Allard MW and Honeycutt RL (1992) Nucleotide sequence variation in the mitochondrial I2S rRNA gene and the phylogeny of African mole-rats (Rodentia: Bathyergidae) Molecular Biology of Evolution 9, 27-40

Bennett NC and Jarvis JUM (1988) The social structure and reproductive biology of colonies of the mole-rat, Cryptomys damarensis (Rodentia, Bathyergidae) Joumal of Mammalogy 69 293-302
Bennett NC, Jarvis JUM and Faulkes CG (in press) Reproductive hormone profiles of three colonies of the eusocial mole-rat, Cryptomys damarensis. In Proceedings of the Second International Conference on Advances in Reproductive Research in Man and Animals, Nairobi, May 1992

Bennett NC, Jarvis JUM, Faulkes CG and Millar RP (1993) LH responses to single doses of exogenous GnRH by freshly captured Damaraland mole-rats, Cryptomys damarensis Joumal of Reproduction and Fertility 99 81-86

Bennett NC, Jarvis JUM, Millar RP, Sasano $\mathrm{H}$ and Ntshinga, KV Reproductive suppression in eusocial Cryptomys damarensis colonies: socially-induced infertility in females. Joumal of Zoology (in press)

Biggers JD, Whitten WK and Whittingham DG (1971) The culture of mouse embryos in vitro. In Methods in Mammalian Embryology Ed. JC. Daniel, Jr Freeman, San Francisco

Brett RA (1991) The population structure of naked mole-rat colonies. In The Biology of the Naked Mole-Rat pp 97-136 Eds PW Sherman, JUM Jarvis and RD Alexander. Princeton University Press, New York

Creel S, Creel N, Wildt DE and Monfort SL (1992) Behavioural and endocrine mechanisms of reproductive suppression in Serengeti dwarf mongooses Animal Behaviour 43 231-245

Faulkes CG (1990) Social Suppression of Reproduction in the Naked Mole-rat Heterocephalus glaber. $\mathrm{PhD}$ Thesis, University of London

Faulkes CG and Abbott DH (1991) Social control of reproduction in breeding and non-breeding male naked mole-rats (Heterocephalus glaber) Journal of Reproduction and Fertility $93 \quad 427-435$

Faulkes CG and Abbott DH (1993) Evidence that primer pheromones do not cause reproductive suppression in male and female naked mole-rats (Heterocephalus glaber) Journal of Reproduction and Fertility 99 225-230

Faulkes CG, Abbott DH and Jarvis JUM (1990a) Social suppression of ovarian cyclicity in captive and wild colonies of naked mole-rats, Heterocephalus glaber Journal of Reproduction and Fertility $88559-568$

Faulkes CG, Abbott DH, Jarvis JUM and Sherriff F (1990b) LH responses of female naked mole-rats, Heterocephalus glaber, to single and multiple doses of exogenous GnRH Journal of Reproduction and Fertility 89 317-323

Faulkes CG, Abbott DH and Jarvis JUM (1991) Social suppression of reproduction in male naked mole-rats, Heterocephalus glaber Journal of Reproduction and Fertility 91 593-604

Fawcett DW, Neaves WB and Flores MN (1973) Comparative observations on intertubular lymphatics and the organization of the interstitial tissue of the mammalian testis Biology of Reproduction 9 500-532

Hickman GC (1979) A live-trap and trapping technique for fossorial mammals South African Journal of Zoology 14 9-12

Jacobs DS, Bennett NC, Jarvis JUM and Crowe TM (1991) The colony structure and dominance hierarchy of the Damaraland mole-rat, Cryptomys damarensis (Rodentia: Bathyergidae), from Namibia Joumal of Zoology 224 553-576

Jarvis JUM (1981) Eusociality in a mammal - cooperative breeding in naked mole-rat Heterocephalus glaber colonies Science 212 571-573

Jarvis JUM (1991) Reproduction. In The Biology of the Naked Mole-Rat pp 384-425 Eds PW Sherman, JUM Jarvis and RD Alexander. Princeton University Press, New York

Jarvis JUM and Bennett NC (1991) Ecology and behaviour of the family Bathyergidae. In The Biology of the Naked Mole-Rat Pp 66-96 Eds PW Sherman, JUM Jarvis and RD Alexander. Princeton University Press, New York

Lacey EA and Sherman PW (1991) Social organization of naked mole-rat (Heterocephalus glaber) colonies: evidence for a division of labour. In The Biology of the Naked Mole-Rat pp 275-336 Eds PW Sherman, JUM Jarvis and RD Alexander. Princeton University Press, New York

Smithers RNH (1983) The Mammals of The Southern African Subregion. Pretoria, University of Pretoria 